\title{
Kapitel 10 \\ Diserzähltes als Erzählfragment
}

\section{Vorbemerkung}

Da sich die Narratologie primär mit expliziten Erzählungen beschäftigt, wird in Diskussionen des Ereignis-Konzepts verständlicherweise in der Regel vorausgesetzt, dass von Aussagen über (in der Textwelt) tatsächlich Geschehenes die Rede ist. Es gibt jedoch durchaus Arbeiten, die sich mit der Frage auseinandersetzen, wie mit Bezügen auf Erwähnungen von Ereignissen umzugehen ist, welche nicht in diese Kategorie fallen. Im Folgenden sollen zwei einflussreiche narratologische Einordnungen - von Gerald Prince und Marie-Laure Ryan - vorgestellt werden sowie im Anschluss eine Adaption aus dem Bereich der neutestamentlichen Wissenschaft.

In einer Auswertung dieser verschiedenen Ansätze wird dann geprüft, wie diese Vorschläge in den Rahmen einer, von Köppe und Kindt repräsentierten, „traditionellen“ Konzeption von Narratologie integriert werden können. Dabei soll insbesondere gezeigt werden, wie von besagten nicht-assertiven Bezügen auf Ereignisse einerseits auf Protoerzählungen andererseits geschlossen werden kann.

Auf diese Weise wird eine heuristisch äußerst ergiebige Perspektive auf die Paulusbriefe eröffnet, die zahlreiche Einzeltexte mit Protoerzählungen in Verbindung bringt - deren Berücksichtigung wiederum die Aussageabsicht der betroffenen Abschnitte insgesamt deutlicher hervortreten lässt.

\section{Das „Diserzählte“}

Die Definition von Narrativität des Narratologen Gerald Prince fokussiert auf den Behauptungs-Charakter von Erzählungen: Als Lesern fällt es uns umso leichter, einen Text als Erzählung zu erkennen, je einfacher er es uns macht, ihn als eine Serie von Behauptungen über das (in der Geschichte) tatsächlich eintretende Geschehen von Ereignissen aufzufassen. ${ }^{1}$ In einem vielbeachteten Aufsatz wendet Prince sich jedoch einem von diesem Prinzip abweichenden, in Erzählwerken und Alltagserzählungen² aber häufig anzutreffenden, Phänomen

1 Prince, Narratology, 149-150. Vgl. zu diesem Themenkomplex auch Kapitel 3, Abschnitt 3.4.3.4. 2 Prince, „Disnarrated,“ 4. 
$\mathrm{zu}$ - dass in diesen nämlich oft Ereignisse eine Rolle spielen, die gerade nicht im besagt typischen Sinn als tatsächlich stattgefunden habend behauptet werden.

Prince unterscheidet zwischen drei verschiedenen Erscheinungsformen dieser Kategorie. Erstens gebe es Ereignisse, die „unerzählbar“ seien (,unnaratable“ oder „nonnarratable“). ${ }^{3}$ Die Gründe für diesen Status könnten unterschiedlich sein. Die Schilderung eines Ereignisses könne etwa gegen ein Gesetz verstoßen - sei es ein soziales oder durch die Grenzen des Genres ${ }^{4}$ auferlegtes. Davon zu unterscheiden sei das Ausbleiben einer Ereignisschilderung aufgrund der fehlenden Kapazität des Erzählers. Schließlich könnte ein Ereignis auch schlicht zu unspektakulär sein, als dass sein Erzählen gerechtfertigt wäre. Was genau „unerzählbar“ ist, hängt natürlich von der jeweiligen Erzählung ab. ${ }^{5}$

Als weitere Ausprägung des diskutierten Phänomens nennt Prince die Ellipse in der Erzählung, also das Auslassen bestimmter Ereignisse, deren Geschehen dennoch angenommen werden kann, weil entweder der Erzähler selbst auf das Fehlen der sprachlichen Repräsentation hinweist oder weil die Chronologie der geschilderten Geschehnisse den Schluss zulässt, dass eine Lücke in der erzählten Handlung besteht. ${ }^{6}$

Der dritte von Prince erwähnte Aspekt stellt in gewisser Weise ein Gegenstück zu diesen „unerzählten“ („unnarated“ oder „nonnarated“) Ereignissen dar. Diesem - als „Diserzähltes“ („disnarrated“) bezeichneten - Bereich ordnet Prince all jene im Text zwar tatsächlich vorliegenden Bezüge auf Ereignisse zu, in welchen aber gerade nicht das Geschehen dieser Ereignisse behauptet wird. ${ }^{7}$

Die Bandbreite der sprachlichen Ausdrucksformen, in welchen diserzählte Ereignisse vorliegen können, gibt Prince wie folgt an: ${ }^{8}$

When I speak of [the disnarrated], I am thus referring to alethic expressions of impossibility or unrealized possibility, deontic expressions of observed prohibition, epistemic expressions of ignorance, ontologic expressions of nonexistence, purely imagined worlds, desired worlds, or intended worlds, unfulfilled expectations, unwarranted beliefs, failed attempts, crushed hopes, suppositions and false calculations, errors and lies, and so forth.

3 Prince, „Disnarrated,“ 1.

4 Vgl. oben (Kapitel 8, Abschnitt 3.3) die Aussagen von Altman zur notwendigen Auslassung von Ereignissen aufgrund der Briefform.

5 Siehe Prince, „Disnarrated,“ 1-2 für unterschiedliche Konstellationen.

6 Prince, „Disnarrated,“ 2. Zum Konzept der ,Ellipse“ siehe auch oben, Kapitel 8, Abschnitt 3.

7 Prince, „Disnarrated,“ 2.

8 Prince, „Disnarrated,“ 3. 


\section{Virtuelle eingebettete Erzählungen}

Mit dieser Kategorie von Prince ist auch verwandt, was Marie-Laure Ryan „virtuelle eingebettete Erzählungen“ nennt. ${ }^{9}$ Diese entstehen, wenn Figuren einer Erzählung durch mentale Akte eine chronologische Sequenz an verbundenen Propositionen hervorbringen. ${ }^{10}$ Dies geschieht etwa, wenn (1) Figuren aufgrund der Ereignisse der erzählten Welt in der Retrospektive einen Zusammenhang aus dem Erlebten konstruieren. ${ }^{11}$ Das Gegenstück bilden (2) mentale Prognosen über die Zukunft, welche entweder (a) passiv den erwartbaren Verlauf der Dinge betreffen oder (b) durch aktives Vorgehen die weitere Entwicklung zu beeinflussen versuchen. Wenn eine Figur einen Wunsch verwirklichen möchte, kommt es zu einem Zusammenspiel dieser verschiedenen Erzählungen:12

The retrospective narrative enables him to evaluate the current situation and to make accurate projections; the passive projection shows him whether or not his desire will be fulfilled by the actions of another individual; and if not, the active projection specifies which steps he should take to realize his goal. When an action is interpreted retrospectively, a fourth narrative comes into play: the actual outcome of the action, which may or may not fall together with the active projection.

Die Miteinbeziehung dieser Art metaphorischer Erzählungen ${ }^{13}$ erlaubt ein komplexes Verständnis von Handlung: „,fabula' is not a linear sequence of events but a bundle of possible stories, some actual and some virtual, whose interaction determines the behavior of characters." 14

Als Leser folgen wir nicht einfach nur der „raw historical sequence, consisting of all the physical states and events in their chronological order," sondern konstruieren auch eine „rationalized sequence." 15 Diese involviert auch die Integration virtueller (d. h. nicht „,aktualisierter,“ also verwirklichter) Narrative - selbst dann, wenn sie im Text nicht explizit durch Verweis auf mentale Akte (Wünsche,

9 Siehe grundlegend Ryan, „Embedded Narratives“ und ausführlicher Ryan, Possible Worlds.

10 Ryan, „Embedded Narratives,“ 323.

11 Ryan, „Embedded Narratives,“ 323: „While the reader reconstrues the fabula on the basis of what the narrator tells him, characters build their own version from what they witness directly and from what is reported to them by speakers they believe reliable.“

12 Ryan, „Embedded Narratives,“ 323.

13 „Metaphorisch“ sind sie, weil nach Ryan, „Embedded Narratives,“ 323 aus folgendem Grund: „For me there need be no speech acts and no discourse but simply a mental representation of the same form as the fabula of which it is a part.“

14 Ryan, „Embedded Narratives,“ 324.

15 Ryan, „Embedded Narratives,“ 322. 
Pläne etc.) ausgedrückt werden, aber dennoch postuliert werden müssen, um das Handeln der Figuren plausibel zu machen. ${ }^{16}$

Mit Princes Kategorie des „Diserzählten“ haben die „virtuellen“ Erzählungen von Ryan gemein, dass sie eben nicht dem sich innerhalb der Erzählung verwirklichenden Geschehen entsprechen. Zugleich gibt es jedoch auch Differenzen zwischen den beiden Konzeptionen: Ryans Ansatz ist sowohl spezifischer (die Quelle des virtuellen Narrativs ist ein mentaler Akt einer Erzählfigur ${ }^{17}$ als auch allgemeiner (es ist nicht notwendig, dass sprachlich auf die nicht verwirklichte Handlung hingewiesen wird). ${ }^{18}$

\section{Alternative Handlungsmöglichkeiten}

Das von Ryan auf dieser Grundlage entwickelte Verfahren einer komplexen Plot-Analyse ist in etwas vereinfachter Form mittlerweile auch als „Spielplan für Handlungen“ in methodisches Lehrmaterial für die neutestamentliche Exegese eingegangen. ${ }^{19}$

Finnern und Rüggemeier legen nahe, zusätzlich zu einer Kette der tatsächlichen Handlungskerne auch Handlungsmöglichkeiten aufzuzeichnen, auf welche „die intendierten Rezipienten ... spekulieren können." ${ }^{20}$ Berücksichtigt man nun die unterschiedlichen Wahrscheinlichkeiten der Entwicklungen und gleicht die verschiedenen möglichen Handlungen mit den tatsächlich realisierten Ereignissen ab, so wird auf diese Weise deutlich, an welcher Stelle der Plot die Erwartungen der Rezipienten sprengt. ${ }^{21}$

Worin Finnern und Rüggemeier freilich von Ryans Vorlage (wohl unbemerkt) abweichen, ist die starke Rezipientenorientierung ihrer Version: Bei Finnern

16 So erklärt Ryan, „Embedded Narrative,“ 329 mit Bezug auf die Parabel von der Krähe und vom Fuchs: „The deceptive plan explains every one of [the fox's] actions and turns the raw physical facts into an intelligible sequence. If a reader ascribes to the fox the intent of finding out how well the crow can sing, he has plainly misunderstood the text: this embedded narrative is inconsistent with the actions of the fox after the cheese falls on the ground. And if the reader has no idea of the plan of the fox, he is simply not trying to understand the text as a story. Embedded narratives thus form necessary components of the fabula, necessary insofar as they are determined by the requirements of intelligibility.“

17 Und nicht etwa ein Verweis auf ,a narrative possibility not chosen by the creator of the textual universe“ (Ryan, Possible Worlds, 167; vgl. S. 168-169). Vgl. Prince, „Disnarrated,“ 4-5.

18 Ryan, Narrative Worlds, 168-169.

19 Finnern und Rüggemeier, Methoden, 219-222.

20 Finnern und Rüggemeier, Methoden, 220.

21 Finnern und Rüggemeier, Methoden, 222. 
und Rüggemeier geht es allgemein um die Anregung der Rezipienten „zur Konstruktion alternativer Handlungsverläufe,"22 während Ryan spezifischer auf die „private narratives“ der Figuren abzielt ${ }^{23}$ und diese gerade in aller Deutlichkeit von den ,projections, expectations, and interpretations produced by the reader“ abgrenzen möchte. ${ }^{24}$

Der von Rüggemeier und Finnern angeregte „Spielplan“ geht daher sowohl über die Konzeption des Diserzählten als auch über diejenige der virtuellen eingebetteten Narrative hinaus: Mit letzteren hat er gemein, dass der Spielplan auch solche nichteingetretenen Ereignisse integriert, deren Nichteintreten im Text nicht explizit erwähnt wird, mit dem Diserzählten verbindet ihn, dass diese Ereignisse nicht wie bei Ryan an die mentalen Zustände der Erzählfiguren geknüpft sind.

\section{Unvollständige Plots?}

Versteht man an Köppe und Kindt angelehnt unter dem ,Plot‘ die chronologische Abfolge und sinnhafte Verknüpfung der zentralen Ereignisse einer Erzählung, dann sind lediglich mögliche oder gar unmögliche Ereignisse definitionsgemäß nicht Bestandteil des Plots. ${ }^{25}$ Erzählwerke in denen bloß potenzielle Handlungsverläufe skizziert werden, weisen dementsprechend nicht mehrere Plots auf,

22 Finnern und Rüggemeier, Methoden, 220.

23 Ryan, Narrative Worlds, 147.

24 Ryan, „Embedded Narratives,“ 330. Hervorhebung nicht im Original. Die genaue Beziehung expliziert sie im Anschluss wie folgt: „The two types of construct resemble each other through the mimetism of their projected relation to the fabula, but they are rooted in different realities: while characters reflect on past and future events from within the narrative universe, the reader does the same from without. The formation by the reader of projections and hypotheses is much less rigidly controlled by the text than the generation of embedded narratives: different readers form different expectations, and anticipating the wrong outcome does not make a reader guilty of misreading. He may in fact have fallen into a trap set up by the narrative discourse itself. When the reader finds his projections and interpretations falsified by the events, he simply discards them in favor of a better representation. These constructs belong to the history of the reading process, not to the history of the narrative universe. On the other hand, when embedded narratives fail to be actualized they remain permanently inscribed as alternative routes on the map of the plot [i. e., as virtual narratives].“

25 Siehe freilich auch bereits Prince, „Disnarrated,“ 4, der zumindest deren Bedeutung abschwächt: „Like the unnaratable and the unnarated, the disnarrated is clearly not essential to narrative.“ 
sondern sind „eher als Erzählungen ohne vollständig ausgestalteten Plot“ zu verstehen. ${ }^{26}$

Vor diesem Hintergrund scheint es zunächst, als bliebe im Rahmen einer narratologischen Analyse nach dem Verständnis von Köppe und Kindt für die von Prince, Ryan und Rüggemeier/Finnern ins Auge gefassten Aspekte wenig Platz. Allerdings gestehen die Autoren an anderer Stelle die Möglichkeit zu, dass eine Binnenerzählung zeitlich auf ihre Rahmenerzählung ${ }^{27}$ nachfolgen kann: „In diesem Fall entwickelt die eingebettete Erzählung eine mögliche Zukunft der Handlung der einbettenden Erzählung. " 28 Die Autoren scheinen also durchaus eine Kategorie des „potenziellen Plots“ zuzulassen, wie auch das von ihnen angeführte Beispiel aus Hölderlins Hyperion zeigt: „Ich fürchte für dich, du hältst das Schicksal dieser Zeiten schwerlich aus. Du wirst noch mancherlei versuchen, wirst - O Gott! Und deine letzte Zufluchtsstätte wird ein Grab sein. “29

Im Folgenden soll ein Vorschlag skizziert werden, welcher einerseits an einem strikten Verständnis des Plots festhält, andererseits jedoch die Bedeutung von Ereignissen mit negativem/unklarem epistemischen/ontologischen Status integriert. Dabei soll gezeigt werden, dass die Kategorie des Protonarrativs eine Integration beider Aspekte erlaubt. Auf diese Weise soll der Tatsache Rechnung getragen werden, dass Bezüge auf Ereignisse auch dann zu einer Imaginationstätigkeit einladen, wenn ihr Eintreten nicht behauptet wird. Zugleich wird auf diese Weise aber vermieden, dass die Fragestellung ganz in den Bereich der Rezipienten verlagert wird. Das Phänomen wird demgegenüber vielmehr als Teil der Technik des Erzählers verstanden. ${ }^{30}$

\section{Fazit: Diserzählte Ereignisse als Erzählfragmente von Protonarrativen}

Jede Aussage, die das Geschehen eines Ereignisses in der Vergangenheit zum Gegenstand hat, kann auch verneint werden. Aussagen über Dinge, die nicht

26 Köppe und Kindt, Erzähltheorie, 111. Hervorhebung nicht im Original. Sind die verschiedenen entworfenen Ereignisfolgen das Produkt einer Figur, dann sei der Akt des Erzählens als Teil des Plots der betreffenden Rahmenhandlung zu sehen.

$27 \mathrm{Zu}$ diesen Kategorien siehe oben, Kapitel 3, Abschnitt 5.2.

28 Köppe und Kindt, Erzähltheorie, 176. Hervorhebung nicht im Original.

29 Hölderlin, Hyperion, 75.

30 Textlinguistisch liegt auch hier die Unterscheidung zwischen der rein rezipientenorientierten Bewirkungsfunktion und die an der Kommunikationsabsicht orientierte Textfunktion zugrunde. Vgl. hierzu Gansel und Jürgens, Textlinguistik, 82-83. Siehe bereits oben, Kapitel 9, Abschnitt 4. 
geschehen sind, eignen sich nun auf den ersten Blick nicht als Grundlage für Erzählungen. Anstatt über eine Sache zu einem bestimmten Zeitpunkt etwas auszusagen, wird lediglich geklärt, was zu diesem Zeitpunkt (bzw. -intervall) nicht der Fall war. Es wird auf diese Weise also gerade keine Geschichte erzählt. Jedoch wird dennoch auf eine bestimmte Erzählung fokussiert - indem diese ausgeschlossen wird: ${ }^{31}$

Mit der Verwendung einer Negation ist immer die Hintergrundannahme gekoppelt, dass im gegebenen Kontext das affirmierte Gegenteil zur Debatte stand, das dann in der Negation explizit zurückgewiesen wird.

Als Beispiel mag Paulus’ Formulierung in Gal 1,16c dienen, wo er versichert „nicht sofort Fleisch und Blut zu Rate gezogen zu haben“ ( $\varepsilon u ̉ \theta \varepsilon ́ \omega \varsigma$ oủ $\pi \rho \circ \sigma \alpha v \varepsilon \theta \varepsilon \dot{\mu} \mu \nu$

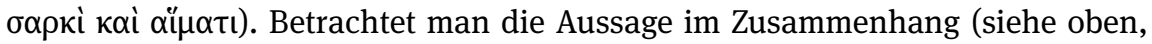
Kapitel 5, Abschnitt 5.1.2 und 5.2.2; vgl. auch Kapitel 8, Abschnitt 5.4), so ist sehr auffällig (vgl. Abb. 25 und Abb. 52), dass die klassisch als „Berufungsgeschichte“ charakterisierte Passage 1,15-16 auf diesen verneinten Hauptsatz hinausläuft, während die eigentliche Beauftragung zur Mission nur als abhängiger Infinitiv

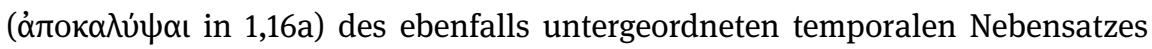
1,15 in Erscheinung tritt (zum elliptischen Erzählen vgl. Kapitel 8, Abschnitt 3).

Es handelt sich hierbei nicht um ein „virtuelles eingebettetes Narrativ,“ denn es wird uns keine weitere Figur vorgestellt, der wir unterstellen könnten, in einem mentalen Akt einen anderen Ablauf imaginiert zu haben, der im Verlauf der Erzählung dann enttäuscht würde. Am ehesten noch könnte man im Final-

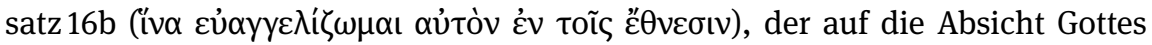
verweist, ein eingebettetes Narrativ sehen - dem Gal 1,16c-17 aber wohl ent- und nicht widerspricht. Ein Gegenbeispiel stellt etwa Apg 9,24 dar: In den Anschlagsplänen der Juden von Damaskus liegt in Ryans Terminologie ein eingebettetes Narrativ vor, das dadurch zunichte (also lediglich „,virtuell“) wird, dass der Plan der Jünger - der wiederum eine passive und aktive Prognose der Geschehnisse voraussetzt - in 9,25 aufgeht.

Ebenso ist fraglich, ob man im Hinblick auf Gal 1,16c unter Rückgriff auf die Kategorien von Finnern und Rüggemeier sagen kann, dass dieser tatsächliche Kern dem virtuellen Kern eines sofortigen Besuchs in Jerusalem eine „Überraschung“ darstellt. ${ }^{32}$ Zwar besteht in der Tat ein Kontrast zur Binnenerzählung 1,13-14, vor dem Hintergrund des Einstiegs von Gal 1,1 und der Versicherung in 1,11-12 wäre

31 Breindl, „Grundbegriffe,“ 123.

32 Zur Differenzierung zwischen leichter Überraschung, Überraschung und provokanter Überraschung siehe Finnern und Rüggemeier, Methoden, 222. 
aber wohl im größeren Kommunikationskontext eher von einer „Bestätigung“ zu sprechen (vgl. Abb. 52 und die dazugehörige Diskussion). Zwar ist durchaus diskussionswürdig, ob hier aus Rezipientenperspektive der tatsächliche Handlungsverlauf der Handlungserwartung folgt. In jedem Fall scheint Paulus großen Wert auf dieses „diserzählte Ereignis“ gelegt zu haben. Und ganz grundsätzlich kann festgehalten werden, dass „kommunikatives Gewicht“ nicht notwendigerweise mit Neuheit einhergehen muss (siehe oben, Kapitel 4, Abschnitt 8.1.3 zur Informationsstruktur). Bei dem von Finnern und Rüggemeier vorgeschlagenen Analyseschritt handelt es sich also um eine durchaus den Kommunikationskontext erhellende Methode. Sie ist aber als weiterführend $\mathrm{zu}$ betrachten und stellt keinen Ersatz für die Wahrnehmung der vom Erzähler vorgenommenen Betonung dar. Diese einfach gänzlich beiseite zu lassen, kommt nicht in Frage, denn dies würde bedeuten, die Erzähltechnik des Erzählers zu ignoriere.

Eine angemessene Einordnung wird dadurch möglich, dass zunächst festge-

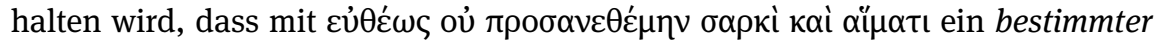
Typ Erzählungen ausgeschlossen wird - nämlich all jene Erzählungen, deren Plots das hier negierte Ereignis aufweisen würden. Auch wenn man nun nicht davon ausgeht, dass Paulus mit dieser verneinenden Versicherung auf eine Erzählung reagiert, die über ihn kursierte, ${ }^{33}$ so wird doch spätestens durch die Aussage selbst dem Leser nahegelegt, sich eine solche Erzählung vorzustellen. Auch wenn das, was nicht geschieht, also nicht Teil des Plots einer Erzählung ist, ist die explizite Erwähnung, dass ein bestimmtes Ereignis nicht eingetreten ist, als Verweis auf eine andere Erzählung zu werten, bzw. genauer als Verweis auf die Handlung einer potenziellen Erzählung.

Zweitrangig ist hierbei die Frage, ob diese Alternativhandlung tatsächlich vom Erzähler der expliziten Erzählung in einem mentalen Akt als Erzählung simuliert wurde, also eine voll (mental) „ausformulierte“ Protoerzählung auf Seiten des Erzählers vorliegt. Auf jeden Fall liegt eine gewisse Narrativisierung eines Ereigniszusammenhangs vor, dessen temporale und sinnhafte Verknüpfung durch den Rezipienten der Kommunikationsintention des Sprechers/Schreibers entspricht. ${ }^{34}$ Dass der Ereigniszusammenhang als voll ausgebildetes Protonarrativ im Bewusstsein des Erzählers vorliegt, ist besonders dann deutlich, wenn eine - in der Regel von anderen geäußerte - Erzählung, die sich im Umlauf befindet, im Text bestritten wird (vgl. etwa unten, Kapitel 11, Abschnitt 2 und Kapitel 12, Abschnitt 2.3.2, zu unten 2. Thess 3,7b-8).

33 Siehe etwa für eine skeptische Einschätzung gegenüber dieser weit verbreiteten Annahme Barclay, „Paul's Story.“

34 Vgl. Kapitel 9, Abschnitt 4. 
Kapitel 12 wird einen ausführlichen Überblick über unterschiedliche Varianten des Diserzählens bieten und auf die verschiedenen sprachlichen Mittel eingehen, von welchen Paulus in konkreten Texten Gebrauch macht. Zunächst soll jedoch in Kapitel 11 die Grundlage gelegt werden, um die zweite Großgruppe an „Erzählfragmenten“ von Protonarrativen, die in dieser Arbeit untersucht werden sollen, einzuführen: Verweise auf zukünftige Ereignisse. 
